\title{
Ruteo y Asignación de Longitud de Onda: Comparación de Algoritmos Genéticos y Templado Simulado
}

\author{
Arturo B. Rodriguez ${ }^{(1)}$, Antonio L. Gutierrez ${ }^{(1)}$, Luis A. Rivera ${ }^{(1)}$ y Leonardo J. Ramirez ${ }^{(2)}$ \\ (1) Universidad de Santiago de Chile, Departamento de Tecnología Industrial, Grupo de Investigación en \\ Nuevas Tecnologías (GINT), Santiago-Chile. (e-mail: arturo.rodriguez@usach.cl, \\ antonio.gutierrez@usach.cl, luis.rivera@usach.cl) \\ (2) Universidad Militar Nueva Granada, División de Desarrollo Tecnológico e Innovación de la Vicerrectoría \\ de Investigaciones, Grupo de Investigación en Telemedicina (TIGUM), Bogotá-Colombia. \\ (e-mail: leonardo.ramirez@unimilitar.edu.co)
}

Recibido Dic. 11, 2013; Aceptado Feb. 19, 2014; Versión final recibida Mar. 5, 2014

\begin{abstract}
Resumen
En este artículo se comparan estudios sobre enrutamiento y asignación de longitud de onda, para mejorar los indicadores de servicio en telecomunicaciones, tales como probabilidad de bloqueo y minimización de la utilización de la red. Las telecomunicaciones modernas están progresivamente sometidas al aumento de la demanda, lo que se traduce en mayores requerimientos en las redes fotónicas de transporte. Este fenómeno obliga a mejorar los sistemas de enrutamiento, con la finalidad de atender mejor a la demanda actual y tener capacidad de atención de la demanda futura. La comparación se realiza entre los algoritmos Templado Simulado (Simulated Annealing) y Algoritmos Genéticos; ambos simulados en la red óptica NSFNET que utiliza multiplexado por división de longitudes de onda, WDM. Los resultados muestran que los algoritmos heurísticos son mejores bajo demanda dinámica de alta carga y que funcionan mejor bajo stress. Se concluye que los algoritmos genéticos dan la mejor solución a la demanda dinámica futura del transporte de datos.
\end{abstract}

Palabras clave: templado simulado, algoritmos genéticos, heuristica, WDM

\section{Routing and Wavelength Assignement: Comparison of Simulated Annealing and Genetics Algortihm}

\begin{abstract}
In this paper compares studies over routing and wavelength assignment for better some services indicators, such as blocking probability and network utilization. In modern telecommunications are gradually subjected to increased demand, which results in higher requirements in photonic transport networks. This phenomenon forces us improve routing systems, in order to better serve current demand and have capacity of attention of future demand. The comparison is made between the algorithms Genetic Algorithms and Simulated Annealing, both simulated in the optical network NSFNET using wavelength division multiplexing WDM. The results showed that heuristic algorithms are better in dynamic demand and high charge, functioning better under stress. It is concluded that genetic algorithms provide the best solution for future demand.
\end{abstract}

Keywords: simulated annealing, genetics algorithm, heuristic, WDM 


\section{INTRODUCCIÓN}

Hoy la discusión alrededor de las redes ópticas se centra en la conmutación (Switching); la cual es preferible dada su alta velocidad de respuesta relativa frente al routing. EI GINT (Grupo de Investigación en Nuevas Tecnologías, USACH) y el TIGUM (Grupo de Investigación en Telemedicina de la Universidad Militar de Nueva Granada) ha observado el acercamiento de las capas superiores de OSI a la capa enlace, lo que a gatillado la reflexión de la permanencia del switching sobre el routing (Li et al., 2007). En cualquier caso, la búsqueda de mejorar el establecimiento de rutas que determinen una conexión rápida y segura, se deberá resolver a través de los diferentes algoritmos que se encuentran en la literatura, como los algoritmos Dijkstra, Floyd-Warshall entre otros, y los algoritmos heurísticos. Sin embargo los algoritmos optimizadores no resultan eficaces frente a la demanda óptica, que requiere de buenas rutas más que de rutas óptimas; esto se debe a la necesidad de disminuir la probabilidad de bloqueo de la red y mejorar la utilización de la red, indicadores muy utilizados en las investigaciones actuales (Zang et al., 2001). El tráfico que soporta una red se clasifica como tráfico estático y dinámico, en redes ópticas de transporte el tráfico fue estático ya que la demanda no era la suficiente para que congestione el sistema; sin embargo las características estáticas del tráfico cambiaron hacia tráfico dinámico y el problema RWA (Routing Wavelength Assignement) tomó relevancia, esto requiere seleccionar una ruta y una longitud de onda para establecer la conexión, bajo la restricción de que no exista cambio de longitud de onda a lo largo de la ruta seleccionada, esto se conoce como CCW (Continuity Constraint Wavelength); esta restricción ayuda a mejorar el retardo en la ruta de la demanda presente (Rodríguez et al., 2011). Pero, se genera un aumento en la utilización de la red aumentando la probabilidad de bloqueo de la demanda futura; ya que los caminos se agotan mientras más rutas se establezcan. Por lo general, este problema se ha resuelto dividiéndolo en dos partes, la primera resuelve la ruta a seguir, basado en minimizar alguna condición pre-establecida (Chu et al., 2003; Zang et al., 2001). La segunda parte resuelve el problema de asignación de longitud de onda. Existen otras propuestas que resuelven el problema desde una perspectiva integral, es decir sin subdividirlo (Rodríguez et al., 2008; Rodríguez et al., 2009). El presente trabajo realiza una comparación de las dos metodologías desde el ámbito heurístico (Rodríguez et al., 2011; Rodríguez et al., 2008), la primera basada en algoritmos genéticos y la segunda basado en Simulated Annelaing; ambos trabajos desarrollados por los equipos GINT (Chile) y TIGUM (Colombia) en contraste con dos trabajos convencionales (Chu et al., 2003; Zang et al., 2001), aportando una nueva estrategia en la solución del problema RWA con la finalidad de buscar una mejora del desempeño algorítmico bajo stress de carga y detectar a través de la comparación de los indicadores, el comportamiento de los algoritmos heurísticos mencionados bajo cargas superiores a los 100 Erlangs.

\section{DESCRIPCIÓN DEL PROBLEMA}

En las redes totalmente ópticas (AON- All Optical Network) los transmitter o transmisores están conectados con cada receptor en todas las longitudes de onda y por lo tanto se requieren tantas señales como longitudes de onda existen (Yoo et al., 2003; Verma et al., 2000). Cuando muchas sesiones utilizan la misma longitud de onda estas señales no se pueden enviar sobre la misma fibra simultáneamente, para evitar las colisiones dentro de la red y por consecuencia el bloqueo de la sesión. Se debe asegurar que si un receptor está en espera de data en una la longitud de onda determinada en un intervalo de tiempo determinado, solo una señal con esa longitud de onda debe llegar al receptor, si 2 o más señales llegan bajo esas condiciones estamos ante un fenómeno llamado contención. La contención se puede evitar aislando las señales espacial y/o temporalmente. Es decir enviando la señal por otro camino y/o en otro tiempo, el aislamiento temporal por lo general es lo que se quiere evitar pues trae consigo latencia.

Una red óptica está formada por OXC (Optical Cross Connect) o Conmutadores ópticos, enlazados por fibras ópticas. Dependiendo de la tecnología de estos conmutadores pueden conmutar fibras, longitudes de onda, bandas de longitudes de onda, canales de tiempo TDM (Skoufis et al., 2003; Mochida et al., 2002). Las rutas ópticas están formadas por enlaces ópticos, los cuales direccionan el haz de luz (datos) a través de la misma longitud de onda según la restricción de continuidad de longitud de onda, sin embargo esta posibilidad se agota cuando la demanda supera el número de sesiones posible; cuando esta restricción no se puede satisfacer es cuando se bloquea la solicitud. Se han desarrollado sistemas de enrutamiento con reutilización de la longitud de onda, donde se permite cambiarla, sin embargo los estudios no muestran cambios significativos en el rendimiento del enrutamiento (Acedo et al., 2006). En la Figura 1, se observan cinco OXC, que forman parte de un sistema de red óptica mayor, las rutas luz se establecen cuando se determinan los pares (Ruta, $\lambda)$ tales como $\left(x-1, x, x+2 / \lambda_{2}\right) ;\left(x-1, x, x+1 / \lambda_{1}\right)$ y $\left(x-2, x, x+1 / \lambda_{3}\right) ;$ al llegar una solicitud de servicio a OXC $C_{x-2}$ cuya solución pasa por los enlaces $(x-2, x, x+2)$ no se podrá asignar la longitud de onda $\lambda_{2}$ ya que está en uso por otra ruta de luz en el enlace $(x, x+2)$; por lo que se deberá utilizar otra longitud de onda o en su defecto buscar una nueva ruta, a este problema se le denomina contención. Sabemos que en la red existe un tráfico actual, y un tráfico por llegar o solicitado; además el tráfico puede ser estático o dinámico, dependiendo de la relación que existe entre el tráfico actual y el que llega (Rodríguez et al., 2008). 


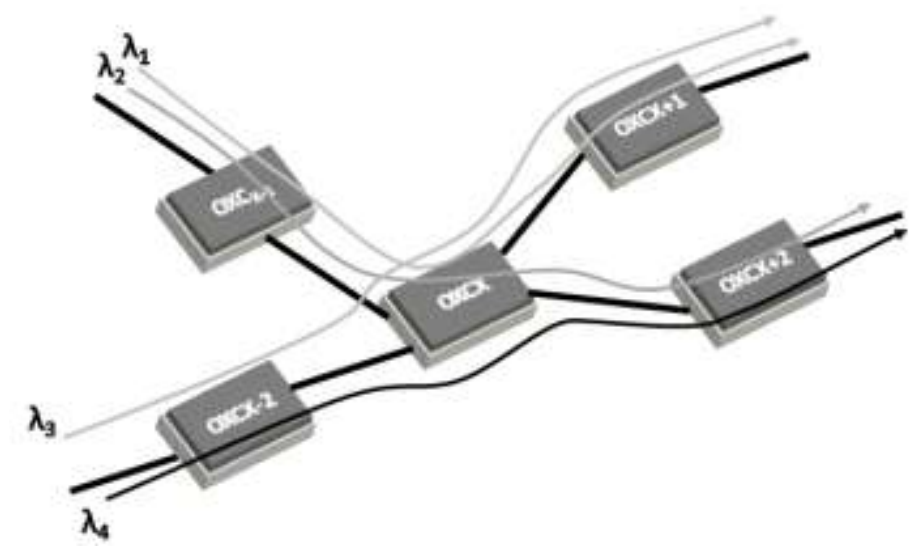

Fig. 1: Ejemplo de contención en Redes ópticas utilizando cuatro longitudes de onda y cinco OXC.

Es dinámico cuando el tiempo de conexión promedio del tráfico actual es mucho menor que el tiempo promedio entre llegadas de solicitud de servicio; este escenario no es optimizable; por lo que se utilizan algoritmos heurísticos; que en su mayoría ofrecen mayores soluciones no necesariamente óptimas; pero esta riqueza apoya mucho cuando se presenta el problema de contención, evitando una nueva ejecución del proceso algorítmico. El problema es establecer las rutas y la asignación de longitud de onda en la red para variados criterios, como mínima probabilidad de bloqueo de solicitudes, baja latencia de transporte en la ruta, bajo nivel de jitter, etc. El problema es conocido como RWA-Routing Wavelength Assignment (enrutamiento y asignación de longitud de onda). Se han ensayado diferentes estrategias para satisfacer la demanda de una red óptica, se han utilizado criterios y algoritmos de optimización. Los criterios de optimización se han basado en, Minimizar la probabilidad de bloqueo de nuevas solicitudes, Minimizar la utilización de longitudes de onda de la red (Ramamurthy et al., 1999), Minimizar el uso de longitudes de onda por enlace (Zang et al., 2000), Minimizar la dispersión en la ruta, Minimizar el ruido ASE en la ruta (Feres et al., 2009) y otros (Balasis et al., 2013; Assis et al., 2010).

Los algoritmos de optimización se pueden clasificar en, convencionales, que son algoritmos que se uutilizan en encaminamiento electrónico o en su defecto solo como valor inicial poblacional para algoritmos heurísticos; tales como: Camino de costos mínimos, Dijkstra, camino de costos incrementales, Bellman Fulkerson caminos de mínimo retardo, Bellman Ford y Programación Lineal entre otros. Heurísticos, que son algoritmos muy utilizados en la búsqueda de buenas rutas y no necesariamente rutas optimas, tales como: Hormiga, Genéticos, Búsqueda Tabú y Simulated Annealing (Tan, 2012; Rodríguez et al., 2010).

El diagrama de la Figura 2, muestra los procesos ubicados en la literatura, para resolver el problema RWA. Como se observa, va desde utilizar estrategias de división del problema hasta resolverlo integralmente, utilizando distintos criterios y algoritmos que permitan obtener una ruta y una longitud de onda; la cual permita transportar la información. Cabe resaltar, que en redes ópticas no necesariamente se busca optimizar, lo que se busca es una ruta que funcione, sin generar un aumento importante de la probabilidad de bloqueo y sin aumento de la red.

\section{DESCRIPCION DE LOS ALGORITMOS}

Los algoritmos comparados, resuelven el problema dividiéndolo en 2 partes e integralmente; para el primer caso se utilizó el algoritmo Simulated Annealing, y para el segundo caso Algoritmos Genéticos, ambos trabajos realizados anteriormente por el GINT del Departamento de Tecnologías Industriales de la Facultad Tecnológica de la Universidad de Santiago de Chile, Chile y el grupo TIGUM de la Universidad Militar de Nueva Granada, Bogotá-Colombia.

El algoritmo Simulated Annealing, utiliza una estrategia basada en el principio de enfriamiento de los materiales, en su paso a lograr estados de cristalización. El trabajo mostrado en (Rodríguez et al., 2011), realiza una simulación del algoritmo entregando buenos resultados sobre la probabilidad de bloqueo y la utilización de la red. El algoritmo utiliza una matriz de rutas candidatas, que son obtenidas aleatoriamente y luego son rotadas por capas a diferentes velocidades la cual disminuye en cada iteración y en cada proceso rotativo se calcula la función de aptitud, que es el costo de la ruta, el objetivo es buscar un costo mínimo dentro del universo de rotación. En el trabajo mencionado, se utilizó la estrategia de dividir, es decir primero calcular la ruta y luego asignarle una longitud de onda, mediante el algoritmo FF (First Fit). 


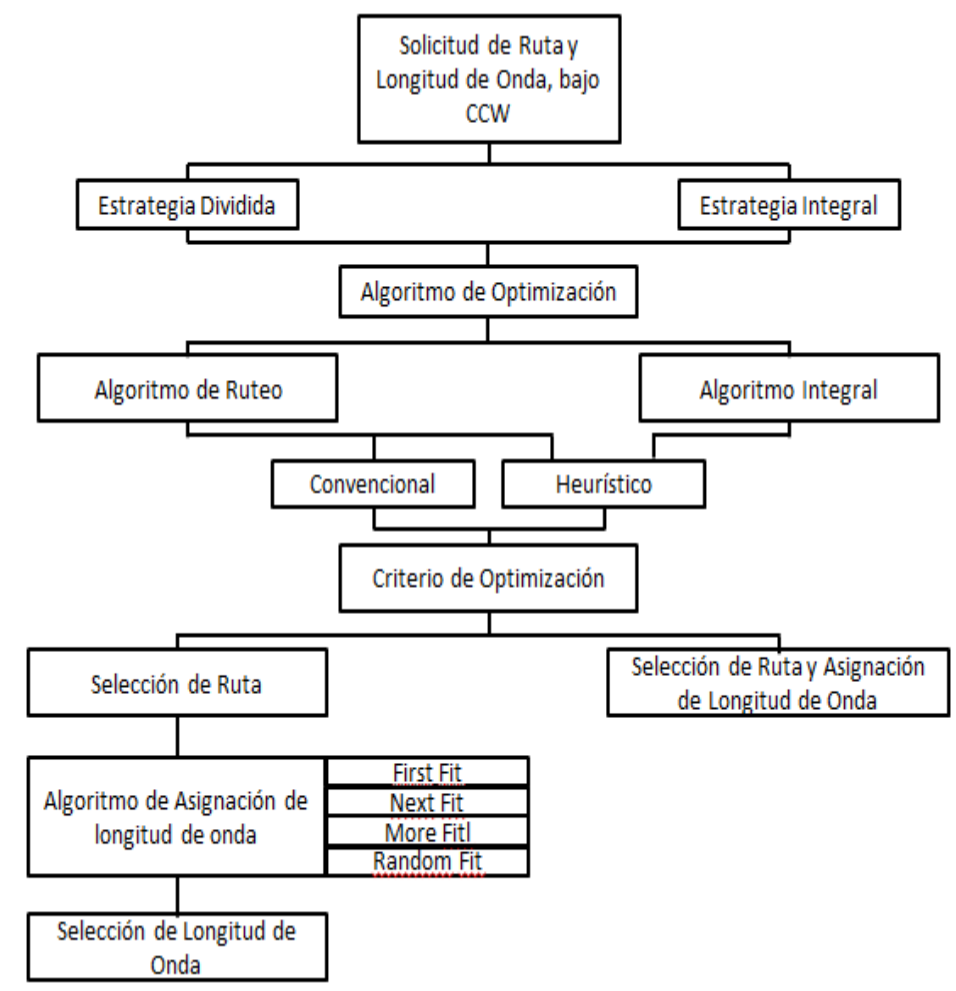

Fig. 2: Estrategias de solución para el problema de enrutamiento y asignación de longitud de onda.

El algoritmo genético, utiliza una estrategia basada en el proceso de evolución biológica basado en su base genética, es una serie de pasos que realizan reproducción, ordenamiento, mutación y mortandad (Valderrama et al., 2006; Rosete et al., 2006; Castrillón et al., 2006). En este caso, se utilizó la estrategia integral, que calcula en un solo proceso algorítmico la ruta y la longitud de onda, con una función de aptitud innovadora. El Grupo de Investigación en Nuevas Tecnologías (GINT), ha realizado ambos trabajos mostrados en (Rodríguez et al., 2011; Rodríguez et al., 2008), la comparación mostrará que los algoritmos heurísticos mejoran el Switching en las Redes WDM, basando su análisis en los indicadores más utilizados en la literatura que son la probabilidad de bloqueo y la utilización de la red.

\section{ESCENARIO DE COMPARACIÓN}

La simulación se comparó con dos trabajos de optimización convencional bajo condiciones de simulación iguales, (Chu et al., 2003; Zang et al., 2001).

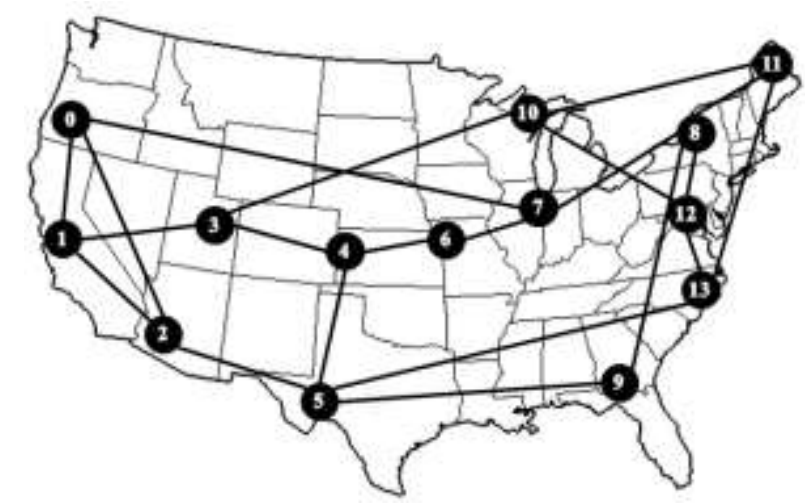

Fig. 3: Red NSFNET utilizada en los cuatro métodos.

La red utilizada para la prueba fue la NSFNET (National Science Foundation Network) que posee 16 nodos y 25 enlaces de fibra óptica (figura 3), los parámetros utilizados fueron similares a los presentados en (Chu et al., 2003), se realizaron comparaciones de la probabilidad de bloqueo y utilización de la red, variando la carga en el intervalo $[0,180]$ con incrementos de 10 Erlangs. El número de conexiones realizadas durante la simulación en ambos escenarios; tanto en Simulated Annealing (SA) como en Algoritmos Genéticos (AG) fue de $10^{8}$ solicitudes de conexión. 


\section{RESULTADOS}

Se realizan comparaciones de las simulaciones entre Simulated Annealing, Algoritmos Genéticos, del Dr. Xiao Wen y del Dr. Hui Zang. Con el indicador de probabilidad de bloqueo, que mide la probabilidad de bloquear las solicitudes de servicio bajo una carga determinada, y el indicador utilización de la red que mide la ocupación de la red bajo una carga determinada. En la Figura 4, se grafica la probabilidad de bloqueo y podemos observar que hasta los 120 Erlangs, el algoritmo genético tiene un mejor comportamiento que Simulated Annealing, después de los 120 Erlangs el algoritmo Simulated Annealing es mejor que el basado en algoritmos genéticos, sin embargo en ningún caso son mejores que el de Hui Zang; se debe observar el excelente comportamiento del algoritmo de Xiao Wen; pero se debe a que se utilizaron 40 longitudes de onda, comparado con las 8 longitudes de onda utilizados por los otros 3 estudios.

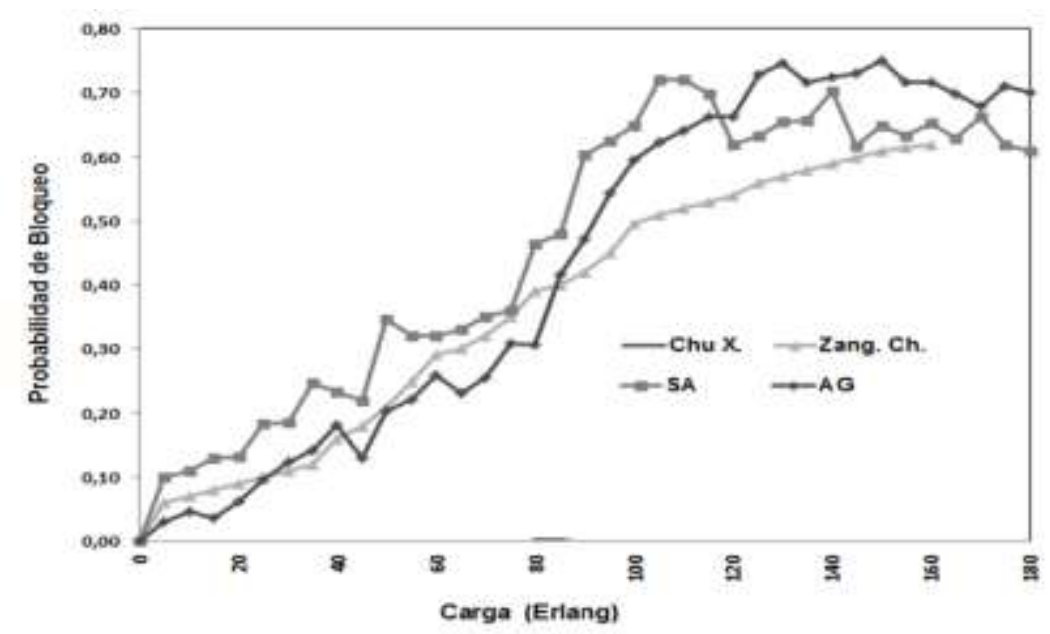

Fig. 4: Comparación de la Probabilidad de Bloqueo en la NSFNET

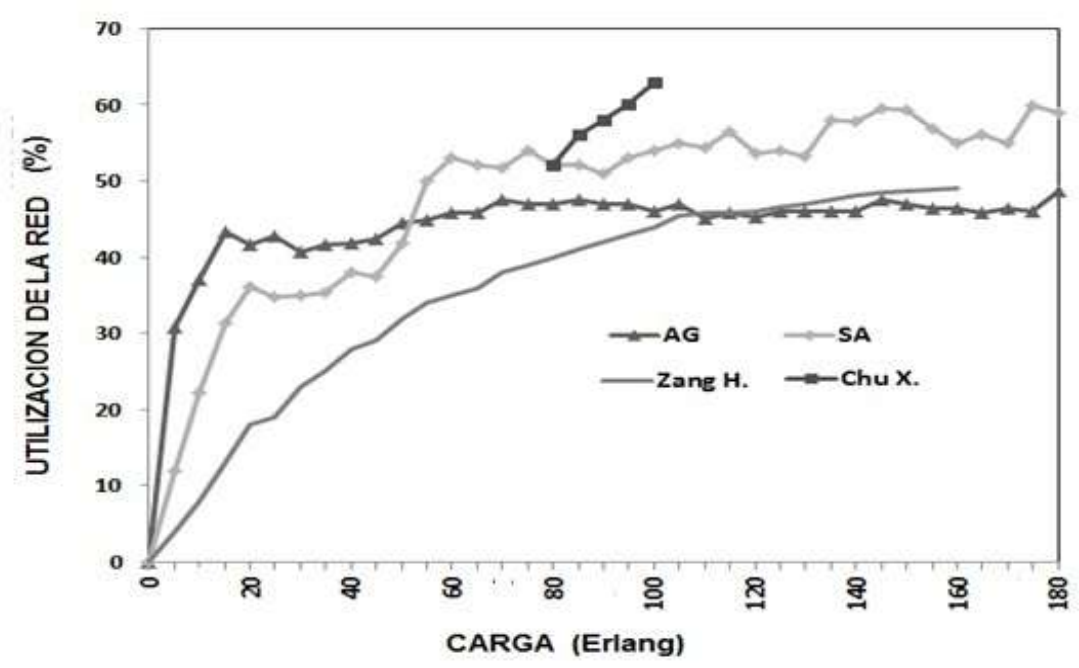

Fig. 5: Comparación de la utilización de red en la NSFNET

En la Figura 5, se grafica la utilización de la red porcentualmente, y puede observar la comparación de la utilización de la red con los trabajos antes mencionados. El algoritmo SA mejora la utilización de la red para cargas inferiores a 50 Erlangs, pero para cargas superiores es el algoritmo AG quien utiliza menos recursos de la red. Pero por debajo de los 110 Erlangs los algortimos SA y AG no son mejores que los de referencia; sin embargo el algoritmo AG es mejor para cargas superiores a los 110 Erlangs, nótese que los trabajos de SA y AG llegan hasta los 180 Erlangs, mientras que los otros trabajos son simulados en intervalos de menor carga de trabajo. 


\section{CONCLUSIONES}

Los trabajos vistos en (Chu et al., 2003; Zang et al., 2001), siempre buscan soluciones óptimas, mientras que los trabajos basados en los algoritmos SA y AG buscan soluciones sin necesidad de recorrer todo el universo posible, de esta manera se ahorra una gran cantidad de tiempo operativo. Por otro, lado, el comportamiento del algoritmo visto en (Chu et al., 2003) tiene una alto consumo de la red aun cuando utiliza cinco veces más longitudes de onda que los trabajos en comparación, lo que genera un problema para satisfacer demanda de carga futura. Esto demuestra que no necesariamente un aumento del número de longitudes de onda mejoraría la utilización de la red. Después de observar los resultados se puede concluir que los algoritmos heurísticos tienen mucho potencial para la demanda futura, debido a que funciona mucho mejor en cargas altas y bajo tráfico dinámico. El tráfico dinámico será muy importante porque permite el uso de la red con un sentido óptimo. El equipo de investigación coincide que será necesario realizar pruebas en redes con reutilización de la longitud de onda, para mejorar la utilización de red y el bloqueo asociado, mientras se desarrollan mejores propuestas.

\section{REFERENCIAS}

Acedo P., Lamela H. and Roda C., Optoelectronic Up-Conversion Using Compact Laterally Mode-Locked Diode Lasers, IEEE Photonics Technology Letters,18, 1888-1890 (2006)

Assis K., Ferreira D.y Giozza W., Hybrid Algorithms for Routing and Assignment Wavelengths in Optical Networks, Latin America Transactions, 8, 214- 220(2010).

Balasis F., Wang X., Xu S. y Tanaka Y., A dynamic physical impairment-aware routing and wavelength assignment scheme for 10/40/100 Gbps mixed line rate wavelength switched optical networks, Advanced Communication Technology (ICACT), 2013 15th International Conference on, 116- 121(2013).

Castrillón O., Sarache W. y Giraldo J., Parámetros Numéricos en el Método de los Algoritmos Genéticos para el Sublimación. Inf. tecnol. [online], 22()1, 83-92(2011).

Feres M. y Trevelin L., RWA algorithm aware of PMD and ASE for all-optical networks, Transparent Optical Networks, 2009. ICTON '09. 11th International Conference on, 1-4 (2009).

Li G., Wang D., Yates J. and R. Doverspike, IP over Optical Cross-Connect Architectures, IEEE Communications Magazine, 45, 34-39 (2007).

Mochida Y., Yamaguchi N. and Ishikawa G., Technology-Oriented Review and Vision of $40 \mathrm{~Gb} / \mathrm{s}$-Based Optical Transport Networks, IEEE Ligthwave Technology, 20, 2272-2281 (2002).

Rodriguez A. y Saavedra F. y Ramirez L., Solución Simultánea Del Enrutamiento Y Asignación De Longitud De Onda en redes WDM Con Algoritmos Genéticos. IEEE COLCOM2008, Bogota, Colombia, (2008).

Rodriguez A. y Saavedra F., Enrutamiento y asignación de longitudes de onda en redes WDM :solución simultánea basada en algoritmos genéticos, IEEE InterconUNSA, Arequipa-Peú, (2009).

Rodríguez A., Saavedra F. y Ramirez L., Simulated Annealing una Propuesta de Solución al Problema RWA en Redes Fotónicas. IEEE InterconUNI, Lima-Peú, (2011).

Rodriguez, A. y Saavedra F., Optimización del Algoritmo Genético para la Solución Integral de Enrutamiento en Redes Fotónicas. Inf. tecnol. [online], 21(3), 125-133(2010) .

Rosete J. y Vega A., Aplicación de Algoritmos Neurogenéticos en la Planeación de las Trayectorias de un Robot Polar. Inf. tecnol. [online], 17(3), 157-165(2006) .

Skoufis C., Sygletos S., Leligou N., Matrakidis C., Pountourakis I. and Stavdas A., Data-Centric Networking Using Multiwavelength Headers/Labels in Packet-Over-WDM Networks: A Comparative Study, IEEE Ligthwave Technology, 21, 2110-2122 (2003).

Tan S., Ant-based Physical Attack and Amplifier Spontaneous Emission-aware routing, Communication Technology (ICCT), 2012 IEEE 14th International Conference on, 650- 653(2012).

Valderrama J. y Faúndez C., Parámetros Numéricos en el Método de los Algoritmos Genéticos para el Sublimación. Inf. tecnol. [online], 17(3), 119-24(2006) .

Verma S., Chaskar H. and Ravikanth R., Optical Burst Switching: A Viable Solution for Terabit IP Backbone, IEEE Network, 14, 48-53 (2000).

Yoo Y. and Sang Kim C., Adaptative Routing Considering the Number of Available Wavelengths im WDM Networks, IEEE Selected Areas in Communications, 21, 1263-1273 (2003).

Zang H. and J. Jue, Dynamic Lightpath Establishment in Wavelength-Routed WDM Networks, IEEE Communications Magazine, 39, 100-108 (2001).

Zang $\mathrm{H}_{\text {., }}$ Jue J., y Mukherjee B., A review of routing and wavelength assignment approaches for wavelength-routed optical WDM networks, Optical Network Magazine, 1, 47-60 (2000). 